\title{
HACIA EL RECONOCIMIENTO DE LAS DESIGUALDADES ENTRE MUJERES Y VARONES EN EL MERCADO LABORAL DEL CHACO. EL COMPORTAMIENTO DE LA SEGREGACION HORIZONTAL EN LOS AÑOS 2001-2010.
}

\author{
Prof. Mgtr. Vilma Lilian Falcón \\ Dpto. de Geografía. Facultad de Humanidades-UNNE \\ Vfalcon_1609@hotmail.com
}

\section{RESUMEN}

Las personas, hombres y mujeres, nacemos al interior de un grupo social que determina qué debemos ser o hacer según nuestra característica biológica, es decir nuestro sexo; esa construcción de parámetros que delimitan el ámbito de actuación y determinan lo masculino y femenino es el resultado de procesos dinámicos en el que intervienen elementos y factores históricos y socioculturales de cada época y lugar. Bajo esos parámetros, hombres y mujeres, desarrollan actividades que, por su construcción y permanencia en el tiempo, se entienden como vinculadas a lo femenino y lo masculino. Un ejemplo de ello es lo que sucede en el espacio del mundo del trabajo, donde se advierte cierto comportamiento diferenciado según se trate del campo de actividad donde varones y mujeres actúan y desarrollan sus labores o de la posición que ambos sexos ocupan en las escalas jerárquicas y salariales entre otros; es decir, un comportamiento que muchas veces expresa situaciones que derivan en desigualdades ocasionando algún tipo de segregación.

En ese contexto, este trabajo pretendemos examinar las condiciones que presentaba el mercado laboral de la provincia del Chaco en los años 2001 y 2010 centrándonos en el concepto de segregación horizontal para observar, a partir del análisis de tres indicadores, las particularidades y su evolución.

\section{PALABRAS CLAVES}

Segregación laboral - Segregación horizontal - Mercado laboral chaqueño - Mujeres Ocupadas Género.

\section{TOWARDS THE RECOGNITION OF THE GAP BETWEEN WOMEN AND MEN IN THE LABOUR MARKET CHACO. BEHAVIOR HORIZONTAL SEGREGATION IN THE YEARS 2001-2010}

\begin{abstract}
SUMMARY
People, men and women are born into a social group that determines what should be or do as our biological feature, ie our sex; Construction of parameters that define the scope and determine masculine and feminine is the result of dynamic processes involved in the elements and historical and cultural factors of every time and place. Under these parameters, men and women develop activities for construction and permanence in time, they are seen as linked to the feminine and the masculine. An example of this is what happens in the space of the working world, where a differentiated behavior is seen depending on whether the field of activity in which men and women act and perform their work or position that both sexes engage in hierarchical scales and salary among others; that is, a behavior that expresses situations often arise in inequalities causing any segregation. In this context, this paper, we examine the conditions presented by the labor market in the province of Chaco in 2001 and 2010 focusing on the concept of horizontal segregation to observe, from the analysis of three indicators, the characteristics and their evolution
\end{abstract}

\section{KEYWORDS}

Occupational segregation - Horizontal segregation - Chaco labor market - Busy Women- Gender

Publicado en formato digital: Prof. Mgtr. Vilma Lilian Falcón. HACIA EL RECONOCIMIENTO DE LAS DESIGUALDADES ENTRE MUJERES Y VARONES EN EL MERCADO LABORAL DEL CHACO. EL COMPORTAMIENTO DE LA SEGREGACION HORIZONTAL EN LOS AÑOS 2001-2010. Revista Geográfica Digital. IGUNNE. Facultad de Humanidades. UNNE. Año 12. № 24. Julio - Diciembre 2015. ISSN 1668-5180 Resistencia, Chaco.

En: http://hum.unne.edu.ar/revistas/geoweb/default.htm 
Revista Geográfica Digital. IGUNNE. Facultad de Humanidades. UNNE. Año 12. № 24 .

Julio - Diciembre 2015. ISSN 1668-5180 Resistencia, Chaco

\section{Introducción}

Las personas, hombres y mujeres, nacemos al interior de un grupo social que determina qué debemos ser o hacer según nuestra característica biológica, es decir nuestro sexo; esa construcción de parámetros que delimitan el ámbito de actuación y determinan lo masculino y femenino es el resultado de procesos dinámicos en el que intervienen elementos y factores históricos y socioculturales de cada época y lugar (1). Bajo esos parámetros, hombres y mujeres, desarrollan actividades que, por su construcción y permanencia en el tiempo, se entienden como vinculadas a lo femenino y lo masculino. Un ejemplo de ello es lo que sucede en el espacio del mundo del trabajo, donde se advierte cierto comportamiento diferenciado según se trate del campo de actividad donde varones y mujeres actúan y desarrollan sus labores o de la posición que ambos sexos ocupan en las escalas jerárquicas y salariales entre otros; es decir, un comportamiento que muchas veces expresa condiciones que pueden derivar en desigualdades.

Entendemos que las condiciones diferenciadas deben ser reconocidas primero para luego evaluar las medidas que permitirán contrarrestarlas y ello nos lleva a considerar la necesidad de incorporar la distinción por sexo en las variables que necesiten ser analizadas, cuestión ineludible, para observar y comprobar si existen y cómo se presentan las condiciones de desigualdad en el mundo laboral, en este caso particular, de la provincia del Chaco. Precisamente, la literatura, cada vez más profusa, nos ilustra acerca del reconocimiento de las condiciones de desigualdad en distintos ámbitos laborales y espacios geográficos; estos estudios analizan y demuestran, a través de diferentes metodologías e indicadores (2) que la segregación por género en el mercado laboral a la vez que persiste también asume características cada vez más complejas. En este sentido, la Organización internacional del Trabajo (OIT) señala que "pese a que la participación femenina en el mercado de trabajo a menudo ha registrado aumentos significativos, la evolución no ha sido pareja entre países y regiones. Sigue habiendo segregación ocupacional horizontal y vertical en función del sexo, y persisten las disparidades salariales por razones de sexo....las mujeres y las niñas siguen ocupándose de una amplísima parte del trabajo asistencial no remunerado; ello limita sus posibilidades de igualdad de oportunidades y de trato ante el empleo en los mercados laborales" (Cfr. OIT, 2014), a la vez que invita a iniciar un proceso de reflexión en el que se evalúe la situación de la mujer en el trabajo en el mundo, en el marco de los logros y las dificultades relacionadas con la consecución de los Objetivos de Desarrollo del Milenio (ODM).

En ese contexto, el aporte de este trabajo es dar continuidad al estudio que venimos realizando en los últimos años (3) al analizar la situación de las mujeres en el mercado laboral en la provincia del Chaco. En esta oportunidad nos centramos en el análisis de tres indicadores frecuentemente utilizados para evaluar las condiciones de segregación de un determinado grupo social; ello, en el marco de una conceptualización de segregación por género -que pretendemos ir construyendo- que entiende que en el mercado laboral existe una concentración de mujeres y hombres en diferentes trabajos que son "característicos" (predominantly) para uno de los dos sexos y ello deriva en la división de trabajos típicamente femeninos y trabajos típicamente masculinos (Cfr. Reskin, B. 1984).

Basados en los resultados obtenidos para el año 2001, con el objetivo de evaluar si hubo variaciones en el comportamiento del mercado laboral desde entonces, incorporamos los resultados del Censo Nacional del año 2010 (4) y, a partir de la comparación, realizamos el análisis de la

Publicado en formato digital: Prof. Mgtr. Vilma Lilian Falcón. HACIA EL RECONOCIMIENTO DE LAS DESIGUALDADES ENTRE MUJERES Y VARONES EN EL MERCADO LABORAL DEL CHACO. EL COMPORTAMIENTO DE LA SEGREGACION HORIZONTAL EN LOS AÑOS 2001-2010. Revista Geográfica Digital. IGUNNE. Facultad de Humanidades. UNNE. Año 12. № 24. Julio - Diciembre 2015. ISSN 1668-5180 Resistencia, Chaco.

En: http://hum.unne.edu.ar/revistas/geoweb/default.htm 
Revista Geográfica Digital. IGUNNE. Facultad de Humanidades. UNNE. Año 12. № 24. Julio - Diciembre 2015. ISSN 1668-5180 Resistencia, Chaco

distribución sectorial de la mano de obra ocupada en la provincia del Chaco tomando para el caso los indicadores de participación, representación y concentración (ver en Anexo los cuadros 4 y 5 )

\section{La segregación en el mercado laboral. Los índices para su medición}

Se dice que existe segregación cuando un grupo de individuos que forma parte del colectivo queda en desigualdad de oportunidades respecto del otro. En el caso del mundo del trabajo se habla de segregación laboral cuando existe una clara distinción entre los sectores de actividad y los puestos de trabajo ocupados por hombres y mujeres, de manera que aparecen como dos colectivos de trabajo, independientes y separados.

Como lo señala la OIT y otros tantos estudios que se ocupan del tema,(5) la segregación ocupacional por razón de género es un fenómeno presente en todos los países, con independencia del nivel de desarrollo económico, e indiferente a la existencia de condicionantes religiosos, culturales y sociales particulares por lo que se presenta como una característica común en todos los mercados de trabajo de todos los países; pero uno de los problemas principales a la hora de evaluar estas condiciones de segregación se da ante la imposibilidad de contar con los datos estadísticos pertinentes, cuestión que también se da en nuestro país y provincia. Es por ello que nos basamos en los datos desagregados por sexo disponibles en los Censos Nacionales mencionados para construir los indicadores que puedan dar cuenta de las desigualdades entre varones y mujeres en el mercado laboral. Al respecto, en este campo, se reconocen al menos los siguientes tipos de segregación:

- La segregación horizontal, que mide el índice de concentración en los sectores o disciplinas ocupacionales.

- La segregación vertical, que afecta a la posición de hombres y mujeres en las jerarquías científicas.

- La segregación financiera, que trata de las diferencias salariales por género.

- La segregación temporal, o la distribución del tiempo dedicado a tares domésticas o de cuidado y a tares científicas.

Dado el objetivo de este trabajo solo nos centramos en la primera de ellas en tanto se refiere a uno de los aspectos de la desigualdad observada entre hombres y mujeres en el empleo: el relacionado con la tendencia que presentan las mujeres a estar ocupadas en diferentes ocupaciones que los hombres, a la vez que quedan formando el grupo en que la mayor parte de las mujeres trabajan en ocupaciones donde los trabajadores son predominantemente mujeres, ocurriendo lo propio con el grupo de varones; es la llamada segregación horizontal.

\section{1.-El comportamiento de los indicadores de segregación horizontal}

\section{1.-La Participación en las Ramas de Actividad Económica}

El primero de los tres indicadores que analizamos nos permite observar cómo se distribuyen varones y mujeres que pertenecen a la Población Económicamente Activa (PEA) ocupada en cada rama de actividad económica. Tanto para el año 2001 como el 2010 hemos realizado el análisis a partir de los datos publicados por el INDEC para la provincia del Chaco tomando en cuenta las ramas de actividad consideradas en cada caso (6), 18 en el primero y 22 en el segundo y, aunque presentan una leve variación en el agrupamiento y denominación, mantuvimos esa distribución en el análisis estadístico, como lo muestra el cuadro $\mathrm{N}^{\circ} 1$ que figura en el Anexo.

Para determinar la distribución porcentual de varones y mujeres en el conjunto de la economía y comprobar el grado de participación que tienen ambos sexos en cada rama o sector de actividad obtuvimos el indicador de participación a partir de la siguiente fórmula:

$$
\text { Participación }=\frac{\text { Población }(V / M) \text { en ocupación i } \times 100}{\text { Total población ocupada en i }}
$$

Publicado en formato digital: Prof. Mgtr. Vilma Lilian Falcón. HACIA EL RECONOCIMIENTO DE LAS DESIGUALDADES ENTRE MUJERES Y VARONES EN EL MERCADO LABORAL DEL CHACO. EL COMPORTAMIENTO DE LA SEGREGACION HORIZONTAL EN LOS AÑOS 2001-2010. Revista Geográfica Digital. IGUNNE. Facultad de Humanidades. UNNE. Año 12. № 24. Julio - Diciembre 2015. ISSN 1668-5180 Resistencia, Chaco.

En: http://hum.unne.edu.ar/revistas/geoweb/default.htm 
Como el objetivo es observar la situación de la mujeres en el conjunto priorizamos el análisis con base en los datos obtenidos para ese grupo, por ello hemos ordenado de mayor a menor los valores correspondientes al grupo Mujeres (Ver en Anexo los cuadros 2 y 3 completos) y, partir de allí obtener algunas conclusiones primarias. En primer lugar, en función de la distribución porcentual en cada rama de actividad, proponemos un recorte basado en los siguientes intervalos que indiquen rubros de:

- Mayor presencia femenina ( $\mathrm{M}=50 \%$ y más)

- Franja de transición/integración ( $M=40 \%$ a $49.99 \%)$

- Mayor presencia masculina ( $M=39.99 \%$ y menos)

\begin{tabular}{|c|c|c|}
\hline Rama de Actividad Agrupada - 2001 & Varones (\%) & Mujeres (\%) \\
\hline $\begin{array}{l}\mathrm{P} \text { - Servicios de hogares privados que contratan servicio } \\
\text { doméstico }\end{array}$ & 9,70 & 90,30 \\
\hline M - Enseñanza & 26,19 & 73,81 \\
\hline N - Servicios sociales y de salud & 35,22 & 64,78 \\
\hline Q - Servicios de organizaciones y órganos extraterritoriales & 50,00 & 50,00 \\
\hline
\end{tabular}

\begin{tabular}{|l|r|r|}
\hline \multicolumn{1}{|c|}{ Rama de actividad Agrupada - 2010 } & Varones (\%) & Mujeres (\%) \\
\hline $\begin{array}{l}\text { (T) Actividades de los hogares como empleadores de personal } \\
\text { doméstico; actividades de los hogares como productores de } \\
\text { bienes o servicios para uso propio }\end{array}$ & 20,14 & \\
\hline (S) Otras actividades de servicios & 26,74 & 79,86 \\
\hline (P) Enseñanza & 35,60 & 73,26 \\
\hline (Q) Salud humana y servicios sociales & 43,52 & 64,40 \\
\hline (I) Alojamiento y servicios de comidas & 47,87 & 56,48 \\
\hline
\end{tabular}

\section{1.a Franja de mayor presencia femenina}

De la comparación entre ambos Censos se observa lo siguiente:

- Las mujeres tienen una participación mayor al $50 \%$ en solo cuatro ramas de actividad en 2001 y en 5 en el año 2010, lo que significa una presencia significativa de ese grupo solo en el 22\% del conjunto de actividades que se desarrollan en la provincia.

- Independientemente de que las ramas de actividad tengan denominación o se agrupen de manera diferentes en ambos censos, el resultado del indicador revela que las mujeres tienen una participación mayor al $50 \%$ en el sector servicios.

- En el marco de dicho sector el rubro (T) Actividades de los hogares como empleadores de personal doméstico; actividades de los hogares como productores de bienes o servicios para uso propio es el segmento donde se manifiesta la mayor presencia femenina en ambos censos. Se trata de actividades relacionadas con las tareas reproductivas del hogar, ya sea que involucre a personas que trabajan para un individuo o familia ó bien para beneficio de su propio hogar (7).

- Respecto del año 2001, en 2010 continúan siendo importantes los valores de participación de las mujeres en áreas como la Educación y la Salud

- Por su parte, evaluando las proporciones correspondientes a 2001 y 2010 se advierte en este último año una mayor participación de varones en las actividades de "servicios" consideradas en el análisis, especialmente en el rubro $(\mathrm{T})$.

Publicado en formato digital: Prof. Mgtr. Vilma Lilian Falcón. HACIA EL RECONOCIMIENTO DE LAS DESIGUALDADES ENTRE MUJERES Y VARONES EN EL MERCADO LABORAL DEL CHACO. EL COMPORTAMIENTO DE LA SEGREGACION HORIZONTAL EN LOS AÑOS 2001-2010. Revista Geográfica Digital. IGUNNE. Facultad de Humanidades. UNNE. Año 12. № 24. Julio - Diciembre 2015. ISSN 1668-5180 Resistencia, Chaco.

En: http://hum.unne.edu.ar/revistas/geoweb/default.htm 
- En 2010 se incorpora el rubro (I) Alojamiento y servicios de comidas, como segmento ocupado mayoritariamente por el grupo femenino (52, 13\%).

\section{1.b Franja de transición y mayor integración}

El intervalo que hemos denominado como franja de transición responde al hecho de haber observado que entre los años 2001 y 2010 al menos una rama de actividad pasó de tener mayor participación masculina a ser ocupada mayoritariamente por mujeres, tal es el caso del rubro I Alojamiento y servicios de comidas al que se hiciera mención en el párrafo precedente. Por otra parte, si tenemos en cuenta el intervalo que estamos considerando ( $M=40 \%$ a $49.99 \%$ ), el grupo de mujeres ha logrado una mayor equiparación con el grupo de varones en actividades referidas a las actividades financieras y seguros, científicas y técnicas, inmobiliarias y comercio. Ello significa que se podría estar ante una tendencia hacia una mayor integración de ambos sexos en estos sectores de actividad económica en la provincia.

Cuadros 2 y 3. Participación de Mujeres y Varones ocupados según rama de actividad 2001-2010 (Franja de transición/integración)

\begin{tabular}{|l|r|r|}
\hline \multicolumn{1}{|c|}{ Rama de Actividad Agrupada - 2001 } & Varones (\%) & \multicolumn{1}{c|}{ Mujeres (\%) } \\
\hline H - Servicios de hotelería y restaurantes & 53,31 & 46,69 \\
\hline Z - Actividades no bien especificadas & 58,53 & 41,47 \\
\hline
\end{tabular}

\begin{tabular}{|l|r|r|}
\hline \multicolumn{1}{|c|}{ Rama de actividad Agrupada - 2010 } & \multicolumn{1}{|c|}{ Varones (\%) } & \multicolumn{1}{|c|}{ Mujeres (\%) } \\
\hline (K) Actividades financieras y de seguros & 51,11 & 48,89 \\
\hline (M) Actividades profesionales, científicas y técnicas & 57,19 & 42,81 \\
\hline $\begin{array}{l}\text { (G) Comercio al por mayor y al por menor; reparación de vehículos } \\
\text { automotores y motocicletas }\end{array}$ & 58,34 & 41,66 \\
\hline (L) Actividades inmobiliarias & 59,31 & 40,69 \\
\hline
\end{tabular}

\section{1.c Franja de mayor presencia masculina (ver cuadro en Anexo)}

En la línea de análisis que venimos realizando, en este intervalo ( $M=39.99 \%$ y menos), la participación de mujeres desciende gradualmente al tiempo que crece la participación masculina mayor al $60 \%$ en las 12 ramas de actividad agrupadas, destacándose con los mayores porcentuales las ocupaciones referidas a la Construcción, Actividades

Primarias (pesca, explotación de minas y canteras, agricultura, ganadería, caza y silvicultura), servicios de transporte e industria manufacturera.

En este caso, en los gráficos 1 y 2 se muestra la

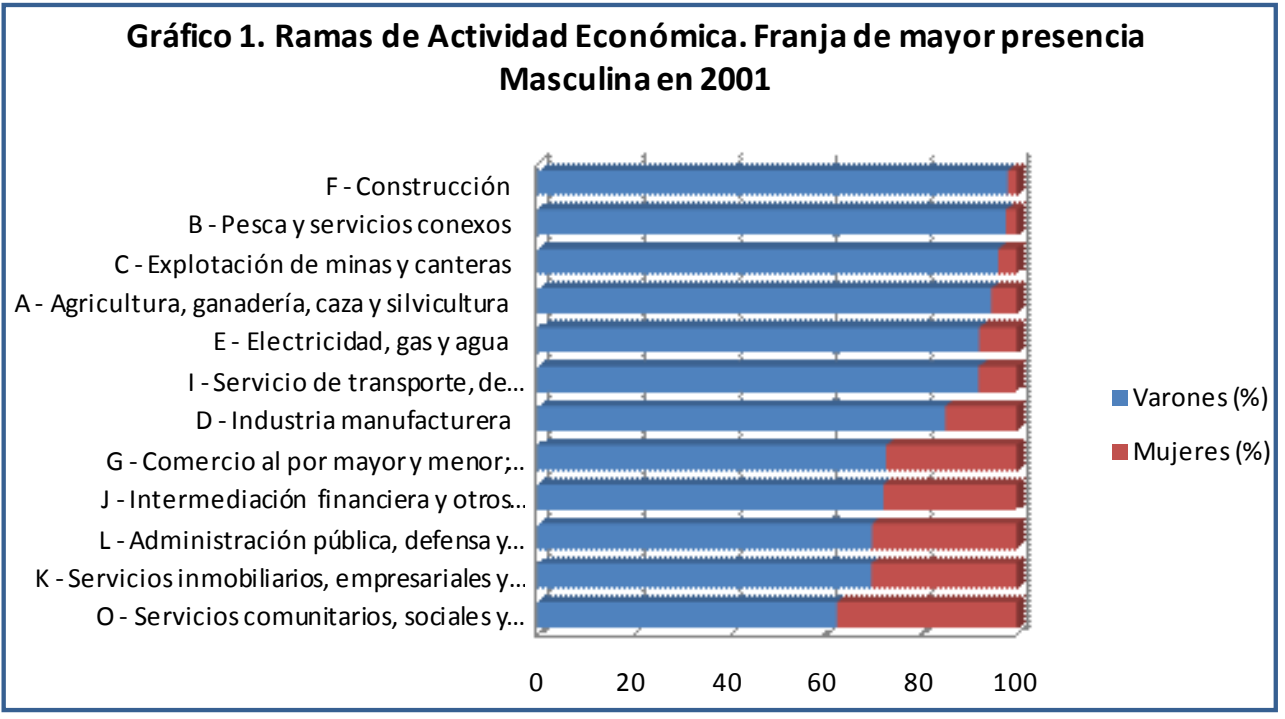

Publicado en formato digital: Prof. Mgtr. Vilma Lilian Falcón. HACIA EL RECONOCIMIENTO DE LAS DESIGUALDADES ENTRE MUJERES Y VARONES EN EL MERCADO LABORAL DEL CHACO. EL COMPORTAMIENTO DE LA SEGREGACION HORIZONTAL EN LOS AÑOS 2001-2010. Revista Geográfica Digital. IGUNNE. Facultad de Humanidades. UNNE. Año 12. № 24. Julio - Diciembre 2015. ISSN 1668-5180 Resistencia, Chaco.

En: http://hum.unne.edu.ar/revistas/geoweb/default.htm 
distribución para ambos años censales a los efectos de su comparación.

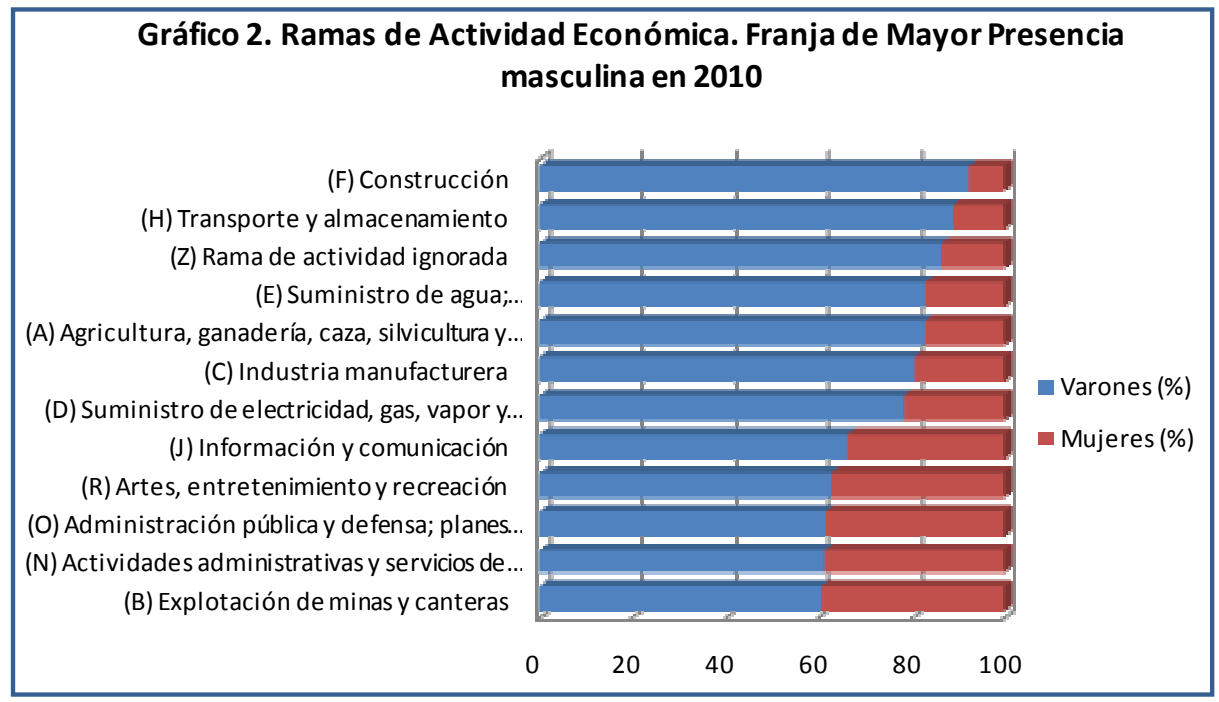

A modo de síntesis de este apartado se puede afirmar que se puede advertir la existencia de ramas de actividad con fuerte presencia de mano de obra femenina y otros sectores que concentran mano de obra masculina. Lo destacable es que, en relación a 2001, para el año 2010 se advierte cierta tendencia hacia una mayor equiparación de la participación de ambos sexos en al menos 5 ramas de actividad tal como lo hemos señalado en los párrafos anteriores.

\section{2.-La Concentración. Comparación del indicador en 2001 y 2010}

El indicador de concentración refleja en que rama de actividad cada grupo concentra la mayor proporción de ocupadas u ocupados, por lo tanto, el resultado surge de aplicar la siguiente fórmula:

\section{Concentración $=\underline{\text { Mujeres/Varones en la ocupación i x } 100}$ Total Mujeres/Varones ocupados}

Como nuestro objetivo es observar en forma comparada los resultados de 2001 y 2010 hemos seleccionado, para cada grupo, las ramas de actividad cuyos porcentajes acumulados concentraran al menos un $70 \%$ (8) de población ocupada en ellas, distinguiendo a ambos sexos en los dos relevamientos censales. Tomamos como base el año 2001 para establecer las posiciones según el valor porcentual que representa a cada sector de actividad; la comparación con el año 2010 solo revela alguna variación de las posiciones de los rubros con mayor concentración de mano de obra femenina pero en ambos años se puede ver que las mujeres ocupadas desarrollan sus actividades relacionadas con el servicio doméstico, la enseñanza, el comercio y en servicios relacionados con la salud, es decir todas correspondiente al sector económico de los servicios.

\begin{tabular}{|c|c|c|c|c|}
\hline \multirow{3}{*}{ Rama de actividad } & \multicolumn{4}{|c|}{ Indice de Concentracion } \\
\hline & \multicolumn{4}{|c|}{ Mujeres } \\
\hline & \multicolumn{2}{|r|}{2001} & \multicolumn{2}{|c|}{2010} \\
\hline $\mathrm{P}$-Servicios de hogares privados que contratan servicio doméstico & $1^{\circ}$ & 24,76 & $3^{\circ}$ & 16,29 \\
\hline M - Enseñanza & $2^{\circ}$ & 21,57 & $2^{\circ}$ & 17,76 \\
\hline G - Comercio al por mayor y menor; reparación de vehículos automotores, mo & $3^{\circ}$ & 13,37 & $1^{\circ}$ & 18,44 \\
\hline L-Administración pública, defensa y seguridad social obligatoria & $4^{\circ}$ & 9,16 & $4^{\circ}$ & 10,89 \\
\hline $\mathrm{N}$ - Servicios sociales y de salud & $5^{\circ}$ & 8,65 & $5^{\circ}$ & 6,67 \\
\hline Corte inmediato superior al $70 \%$ & & 77,51 & & 70,05 \\
\hline
\end{tabular}

Publicado en formato digital: Prof. Mgtr. Vilma Lilian Falcón. HACIA EL RECONOCIMIENTO DE LAS DESIGUALDADES ENTRE MUJERES Y VARONES EN EL MERCADO LABORAL DEL CHACO. EL COMPORTAMIENTO DE LA SEGREGACION HORIZONTAL EN LOS AÑOS 2001-2010. Revista Geográfica Digital. IGUNNE. Facultad de Humanidades. UNNE. Año 12. № 24. Julio - Diciembre 2015. ISSN 1668-5180 Resistencia, Chaco.

En: http://hum.unne.edu.ar/revistas/geoweb/default.htm 
Revista Geográfica Digital. IGUNNE. Facultad de Humanidades. UNNE. Año 12. № 24. Julio - Diciembre 2015. ISSN 1668-5180 Resistencia, Chaco

\begin{tabular}{|c|c|c|c|c|}
\hline & \multicolumn{4}{|c|}{ Indice de Concentracion } \\
\hline \multirow[t]{2}{*}{ Rama de actividad } & \multicolumn{4}{|c|}{ Varones } \\
\hline & \multicolumn{2}{|c|}{2001} & \multicolumn{2}{|c|}{2010} \\
\hline A - Agricultura, ganadería, caza y silvicultura & $1^{\circ}$ & 26,02 & $2^{\circ}$ & 14,45 \\
\hline G - Comercio al por mayor y menor; reparación de vehículos automotores, mo & $2^{\circ}$ & 17,76 & $1^{\circ}$ & 16,1 \\
\hline L - Administración pública, defensa y seguridad social obligatoria & $3^{\circ}$ & 10,53 & $5^{\circ}$ & 10,92 \\
\hline D - Industria manufacturera & $4^{\circ}$ & 10,08 & $3^{\circ}$ & 11,95 \\
\hline F - Construcción & $5^{\circ}$ & 9,17 & $4^{\circ}$ & 11,01 \\
\hline I-Servicio de transporte, de almacenamiento y de comunicaciones & & & $6^{\circ}$ & 6,34 \\
\hline Corte inmediato superior al $70 \%$ & & 73,57 & & 70,77 \\
\hline
\end{tabular}

Del mismo modo, para el caso del grupo varones se observa una concentración de $70 \%$ y más de la mano de obra ocupada en pocas ramas de actividad (5 en 2001) y con solo una incorporación en 2010, tal el caso del sector de servicios de transporte, almacenamiento y comunicaciones. De todos modos, aún cuando en cantidad se asemeja al grupo de mujeres, en este último caso los rubros son más diversificados ya que se encuentran asociados a los sectores económicos primario, secundario y terciario. En forma gráfica se muestra, para el año 2010, la distribución de los porcentajes acumulados que representan la concentración de población ocupada según la cantidad de ramas de actividad.

Grafico 4. Concentración de Varones Ocupados en Ramas de Actividad Económica (Porcentaje Acumulado ). 2010
Gráfico 5. Concentración de Mujeres Ocupadas en Ramas de Actividad Económica

(Porcentaje Acumulado ). 2010
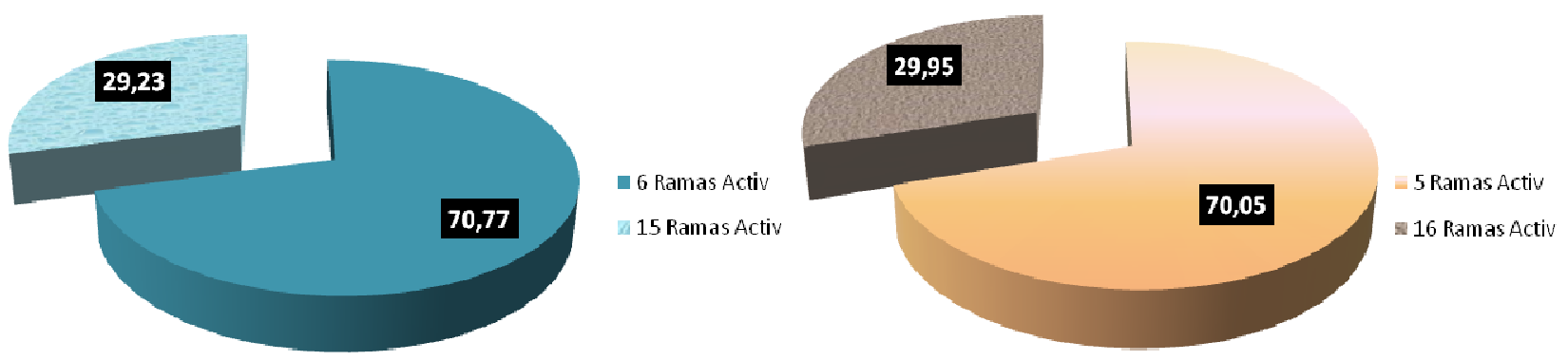

\section{3.- La Representación. Comparación del indicador en 2001 y 2010}

Para observar otras particularidades relacionadas con las presencias femeninas y/o masculinas en determinadas actividades y comprobar con mayor precisión si algunas ramas de actividad pueden considerarse "integradas" por ambos sexos, nos valemos de un indicador que intenta medir la representación de mujeres y varones en los distintos grupos de actividad económica y en los dos años censales.

\section{3.a.- El indicador de Representación.}

Este indicador hace referencia a la relación entre el porcentaje de Mujeres o Varones en una determinada actividad respecto del porcentaje total de Mujeres/Varones en el empleo total, es decir sobre la participación en el total de ocupaciones en la provincia que para 2001 es de $66.68 \%$ para los Varones y de $33.32 \%$ para las Mujeres y, para 2010, es de $61.59 \%$ y $38.41 \%$ respectivamente.

En la fórmula la Representación $=\%$ de Población $(\mathrm{V} / \mathrm{M})$ en ocupación $\mathrm{i}$

$\%$ de población (V/M) en empleo Total

Publicado en formato digital: Prof. Mgtr. Vilma Lilian Falcón. HACIA EL RECONOCIMIENTO DE LAS DESIGUALDADES ENTRE MUJERES Y VARONES EN EL MERCADO LABORAL DEL CHACO. EL COMPORTAMIENTO DE LA SEGREGACION HORIZONTAL EN LOS AÑOS 2001-2010. Revista Geográfica Digital. IGUNNE. Facultad de Humanidades. UNNE. Año 12. № 24. Julio - Diciembre 2015. ISSN 1668-5180 Resistencia, Chaco.

En: http://hum.unne.edu.ar/revistas/geoweb/default.htm 
Siguiendo con el objetivo de observar el comportamiento de la ocupación femenina en el mercado laboral chaqueño, analizamos el resultado desde ese punto de vista; así, si el valor obtenido para la representación en una ocupación determinada es mayor que 1, significaría que las mujeres están sobre-representadas en esa ocupación, en el sentido de que su participación en esa ocupación supera a la participación media de las mujeres en el empleo total de la economía. Por el contrario, cuando se obtiene un valor para la representación que es menor que 1, entonces se dice que las mujeres están sub-representadas en ella.

En el cuadro № 5 se muestran los resultados del cálculo del indicador para el conjunto de categorías para los dos años censales.

Cuadro $\mathbf{N}^{\circ}$ 5. Representación de Mujeres y Varones ocupados según rama de actividad 2001

\begin{tabular}{|l|r|r|}
\hline \multicolumn{2}{|c|}{ Índice de Representación } & \multicolumn{1}{|c|}{ Rama de Actividad Agrupada - 2001 } \\
\hline \multicolumn{2}{|c|}{ Varones } & Mujeres \\
\hline P - Servicios de hogares privados que contratan servicio doméstico & 0,15 & 2,71 \\
\hline M - Enseñanza & 0,39 & 2,22 \\
\hline N - Servicios sociales y de salud & 0,53 & 1,94 \\
\hline Q - Servicios de organizaciones y órganos extraterritoriales & 0,75 & 1,50 \\
\hline H - Servicios de hotelería y restaurantes & 0,80 & 1,40 \\
\hline Z - Actividades no bien especificadas & 0,88 & 1,24 \\
\hline O - Servicios comunitarios, sociales y personales n.c.p. & 0,94 & 1,13 \\
\hline K - Servicios inmobiliarios, empresariales y de alquiler & 1,05 & 0,91 \\
\hline L - Administración pública, defensa y seguridad social obligatoria & 1,05 & 0,91 \\
\hline J - Intermediación financiera y otros servicios financieros & 1,08 & 0,84 \\
\hline G - Comercio al por mayor y menor; reparación de vehículos automotores, etc & 1,09 & 0,82 \\
\hline D - Industria manufacturera & 1,28 & 0,45 \\
\hline I - Servicio de transporte, de almacenamiento y de comunicaciones & 1,38 & 0,24 \\
\hline E - Electricidad, gas y agua & 1,38 & 0,24 \\
\hline A - Agricultura, ganadería, caza y silvicultura & 1,42 & 0,16 \\
\hline C - Explotación de minas y canteras & 1,44 & 0,12 \\
\hline B - Pesca y servicios conexos & 1,47 & 0,07 \\
\hline F - Construcción & 1,47 & 0,06 \\
\hline
\end{tabular}

Como se puede ver en el cuadro № 5, para el año 2001, los valores de representación superiores a 1 se dan en actividades relacionadas con los servicios (comunitarios, sociales, de hotelería y restaurantes, de salud, enseñanza y servicios de hogares privados que contrata servicio doméstico), quedando claramente visible una sobre-representación femenina en actividades relacionadas con los servicios domésticos y la enseñanza. Por su parte, los valores que asume la representación masculina no son tan extremos y, además, se distribuyen en un mayor número de actividades, lo que estaría indicando que el grueso de población femenina ocupada se concentra en un número menor de ocupaciones.

Por su parte, observando los resultados del cuadro correspondiente al año 2010, se puede advertir un comportamiento que no es muy diferente en cuanto a las ramas de actividad donde se da la mayor representación femenina sino en la distribución global de los valores. En función de ello señalamos las siguientes apreciaciones:

- Respecto de 2001, en 2010 las ramas de actividad en la que las mujeres se encuentran sobre representadas aumentaron en cantidad, aunque se mantienen en los primeros

Publicado en formato digital: Prof. Mgtr. Vilma Lilian Falcón. HACIA EL RECONOCIMIENTO DE LAS DESIGUALDADES ENTRE MUJERES Y VARONES EN EL MERCADO LABORAL DEL CHACO. EL COMPORTAMIENTO DE LA SEGREGACION HORIZONTAL EN LOS AÑOS 2001-2010. Revista Geográfica Digital. IGUNNE. Facultad de Humanidades. UNNE. Año 12. № 24. Julio - Diciembre 2015. ISSN 1668-5180 Resistencia, Chaco.

En: http://hum.unne.edu.ar/revistas/geoweb/default.htm 
Revista Geográfica Digital. IGUNNE. Facultad de Humanidades. UNNE. Año 12. № 24. Julio - Diciembre 2015. ISSN 1668-5180 Resistencia, Chaco

lugares la sobre-representación de las mujeres en las mismas ramas de actividad específicas (servicios en los hogares, enseñanza, servicios de salud);

\begin{tabular}{|l|r|r|}
\hline \multicolumn{2}{|c|}{ Índice de Representación } \\
\hline \multicolumn{1}{|c|}{ Rama de actividad Agrupada - 2010 } & Varones & \multicolumn{1}{|c|}{ Mujeres } \\
\hline $\begin{array}{l}\text { (T) Actividades de los hogares como empleadores de personal doméstico; } \\
\text { actividades de los hogares como productores de bienes o servicios para uso propio }\end{array}$ & 0,33 & 2,08 \\
\hline (S) Otras actividades de servicios & 0,43 & 1,91 \\
\hline (P) Enseñanza & 0,58 & 1,68 \\
\hline (Q) Salud humana y servicios sociales & 0,71 & 1,47 \\
\hline (I) Alojamiento y servicios de comidas & 0,78 & 1,36 \\
\hline (K) Actividades financieras y de seguros & 0,83 & 1,27 \\
\hline (M) Actividades profesionales, científicas y técnicas & 0,93 & 1,11 \\
\hline (G) Comercio al por mayor y al por menor; reparación de vehículos automot y motoci & 0,95 & 1,08 \\
\hline (L) Actividades inmobiliarias & 0,96 & 1,06 \\
\hline (B) Explotación de minas y canteras & 0,99 & 1,02 \\
\hline (N) Actividades administrativas y servicios de apoyo & 1,00 & 1,00 \\
\hline (O) Administración pública y defensa; planes de seguro social obligatorio & 1,00 & 1,00 \\
\hline (R) Artes, entretenimiento y recreación & 1,02 & 0,97 \\
\hline (J) Información y comunicación & 1,08 & 0,88 \\
\hline (D) Suministro de electricidad, gas, vapor y aire acondicionado & 1,27 & 0,57 \\
\hline (C) Industria manufacturera & 1,31 & 0,51 \\
\hline (A) Agricultura, ganadería, caza, silvicultura y pesca & 1,34 & 0,45 \\
\hline (E) Suministro de agua; alcantarillado, gestión de desechos y actividades de & 1,34 & 0,45 \\
\hline saneamiento & 1,40 & 0,36 \\
\hline (Z) Rama de actividad ignorada & 1,44 & 0,29 \\
\hline (H) Transporte y almacenamiento & 1,50 & 0,21 \\
\hline (F) Construcción & & \\
\hline
\end{tabular}

- La rama de actividad que hace referencia a los servicios en los hogares se mantiene con un índice muy elevado de sobre representación y, en el caso de las actividades relacionadas con la enseñanza se nota un leve aumento en la representación en el grupo de varones.

- Otra situación destacable es que en 2010 el indicador es igual a 1 en dos actividades (administrativas y servicios de apoyo y en la administración pública y defensa; planes de seguro social) lo que estaría revelando una equiparación de ambos grupos en la ocupación en esos rubros.

- En cuanto la sub representación de la mano de obra femenina se observa que persiste en la mayoría de las ramas de actividades consideradas en 2001 aunque las posiciones, reveladas por los valores del índice, se distribuyen de otra manera.

- Por último, la rama de la construcción da cuenta de una mayor sobre-representación masculina y, en consecuencia, es donde la mano de obra femenina está más subrepresentada.

\section{2- Caracterización de los sectores de Actividad Económica según la Representación}

Una manera de determinar si un sector de actividad se encuentra "feminizado", "masculinizado" o "integrado" requiere en primer lugar seleccionar una variable que permita agruparlas y posteriormente adoptar un criterio de agrupamiento (9). En este caso, tal como le hemos

Publicado en formato digital: Prof. Mgtr. Vilma Lilian Falcón. HACIA EL RECONOCIMIENTO DE LAS DESIGUALDADES ENTRE MUJERES Y VARONES EN EL MERCADO LABORAL DEL CHACO. EL COMPORTAMIENTO DE LA SEGREGACION HORIZONTAL EN LOS AÑOS 2001-2010. Revista Geográfica Digital. IGUNNE. Facultad de Humanidades. UNNE. Año 12. № 24. Julio - Diciembre 2015. ISSN 1668-5180 Resistencia, Chaco.

En: http://hum.unne.edu.ar/revistas/geoweb/default.htm 
hecho para el año 2001 (Cfr. Falcón, V. 2011) hemos seleccionado a la variable Representación y a partir de su resultado definimos un umbral ajustándonos a la propuesta que realizan Cáceres Ruiz, J. y otros (2004) quienes adoptan el valor de 1.25 para determinar las siguientes agrupaciones:

- ocupaciones femeninas son aquellas en las que la representación de las mujeres es mayor a 1,25 ;

- ocupaciones masculinas son aquellas en las que la representación de los hombres es mayor a 1,25

- ocupaciones integradas en términos de género son consideradas aquellas en las que la representación de las mujeres/hombres está comprendida entre 1 y 1,25.

Con base en esta clasificación hemos realizado en el cuadro № 6 la caracterización de los sectores de actividad económica de la provincia del Chaco para el año 2010, quedando claramente expuesto que el mercado laboral de la provincia se mantiene -en el período considerado- con las mismas características en la distribución de la mano de obra femenina y masculina en ciertos sectores o ramas de actividad: mujeres en el sector terciario o de servicios; varones en sectores más diversificados y finalmente un leve aumento de las ramas de actividad en la que se da una mayor equiparación entre varones y mujeres pero que corresponden mayoritariamente al sector terciario.

\section{Cuadro № 6. Distribución de la mano de obra ocupada según agrupamiento del Índice de Representación}

\begin{tabular}{|c|c|c|}
\hline $\begin{array}{l}\text { Ocupaciones Femeninas } \\
\qquad(>1.25)\end{array}$ & $\begin{array}{c}\text { Ocupaciones Masculinas } \\
\qquad(>1.25)\end{array}$ & $\begin{array}{c}\text { Ocupaciones Integradas } \\
\text { (1 a 1.25) }\end{array}$ \\
\hline $\begin{array}{l}\text { (T) Actividades de los hogares como } \\
\text { empleadores de personal doméstico; } \\
\text { actividades de los hogares como } \\
\text { productores de bienes o servicios para } \\
\text { uso propio }\end{array}$ & (F) Construcción & $\begin{array}{l}\text { (M) Actividades profesionales, } \\
\text { científicas y técnicas }\end{array}$ \\
\hline (S) Otras actividades de servicios & $\begin{array}{l}\text { (H) Transporte y } \\
\text { almacenamiento }\end{array}$ & $\begin{array}{l}\text { (G) Comercio al por mayor y al } \\
\text { por menor; reparación de } \\
\text { vehículos automot y motoci }\end{array}$ \\
\hline (P) Enseñanza & (Z) Rama de actividad ignorada & (L) Actividades inmobiliarias \\
\hline (Q) Salud humana y servicios sociales & $\begin{array}{c}\text { (E) Suministro de agua; } \\
\text { alcantarillado, gestión de } \\
\text { desechos y actividades de } \\
\text { saneamiento }\end{array}$ & $\begin{array}{l}\text { (B) Explotación de minas y } \\
\text { canteras }\end{array}$ \\
\hline (I) Alojamiento y servicios de comidas & $\begin{array}{l}\text { (A) Agricultura, ganadería, } \\
\text { caza, silvicultura y pesca }\end{array}$ & $\begin{array}{c}\text { (N) Actividades administrativas } \\
\text { y servicios de apoyo }\end{array}$ \\
\hline \multirow[t]{3}{*}{$\begin{array}{l}\text { (K) Actividades financieras y de } \\
\text { seguros }\end{array}$} & (C) Industria manufacturera & $\begin{array}{c}\text { (O) Administración pública y } \\
\text { defensa; planes de seguro } \\
\text { social obligatorio }\end{array}$ \\
\hline & $\begin{array}{l}\text { D) Suministro de electricidad, } \\
\text { gas, vapor y aire } \\
\text { acondicionado }\end{array}$ & $\begin{array}{l}\text { (R) Artes, entretenimiento y } \\
\text { recreación }\end{array}$ \\
\hline & & (J) Información y comunicación \\
\hline
\end{tabular}

\section{A modo de conclusión}

Sabemos que determinar la existencia o no de segregación por género en el mundo laboral exige mucho más que analizar unos datos desagregados por sexo pero, como lo señaláramos al iniciar este trabajo, entendemos que es absolutamente necesario esa primera aproximación para

Publicado en formato digital: Prof. Mgtr. Vilma Lilian Falcón. HACIA EL RECONOCIMIENTO DE LAS DESIGUALDADES ENTRE MUJERES Y VARONES EN EL MERCADO LABORAL DEL CHACO. EL COMPORTAMIENTO DE LA SEGREGACION HORIZONTAL EN LOS AÑOS 2001-2010. Revista Geográfica Digital. IGUNNE. Facultad de Humanidades. UNNE. Año 12. № 24. Julio - Diciembre 2015. ISSN 1668-5180 Resistencia, Chaco.

En: http://hum.unne.edu.ar/revistas/geoweb/default.htm 
poder determinar cómo se estructura el mercado de trabajo en la provincia, cómo se distribuyen varones y mujeres en los distintos sectores de actividad económica para luego iniciar un proceso de reconocimiento de las condiciones diferenciadas que podrían derivar en segregación. Precisamente, tal como nos hemos propuesto, en este trabajo nos ocupamos de revelar esas condiciones diferenciadas presentes en el mercado laboral de la provincia a partir del análisis de la segregación horizontal, es decir de cómo se distribuyen varones y mujeres en los sectores de actividad económica.

Considerando el resultado de los tres indicadores seleccionados -participación, concentración y representación- se advierte que existe una polarización de la mano de obra femenina en un segmento reducido de actividades económicas relacionadas con la prestación de servicios, tanto para el año 2001 como para el 2010, es decir que prácticamente no hubo variaciones en ese sentido, por lo tanto las mujeres se siguen desempeñando en tareas relacionadas con el servicio en los hogares, en la educación, en los servicios sociales y de salud; en servicios sociales y servicios de hotelería y restaurantes, es decir que la mano de obra femenina se concentra en un $28 \%$ del total de las actividades económicas de la provincia, apareciendo en algunas de ellas con una excesiva sobrerepresentación. Del mismo modo, de acuerdo a lo que muestra el indicador de representación, la mano de obra masculina también muestra cierta concentración en un numero levemente superior de ramas de actividad (33.3\% del total), aunque la particularidad de este caso es que son rubros más diversificados en su correspondencia con los sectores primarios, secundarios y terciarios.

Por su parte, las ocupaciones integradas o mixtas quedan definidas como aquellas que muestran un mayor equilibrio en cuanto a la presencia de mano de obra femenina o masculina y responden a actividades relacionadas con los servicios comunitarios, sociales y personales, servicios inmobiliarios, administración pública, defensa y seguridad social obligatoria, servicios financieros, y comercio entre otros. Es en este grupo de actividades donde se observa la mayor variación entre 2001 y 2010 dado que la representación indica que se pasó de un $22 \%$ a $38 \%$ de ramas de actividades ocupadas por varones y mujeres, hecho que refleja una mayor equiparación entre ambos sexos.

Finalmente podemos concluir que la situación del mercado laboral en nuestra provincia no se aleja del comportamiento general del mercado laboral de otras regiones del mundo; numerosos estudios indican que si bien se registra un leve aumento de la participación de las mujeres en la economía se mantiene su concentración en unos pocos sectores de actividad, hecho que demuestra la persistencia de una segregación horizontal.

\footnotetext{
Notas

${ }^{1}$ Es la llamada construcción social de género y es a través de la cual se potencian y originan las desigualdades de género, es decir la subordinación de uno de ellos, frente al dominio y poder del otro género.

${ }^{2}$ Véase por ejemplo a: López, Guadalupe (2006); Albert, Rocío y otros (2005); Herrero, María José; Maté García, Jorge y otros (2007); Cáceres Ruiz, Juan I. y otros (2004).

${ }^{3}$ La segregación laboral es un tema que nos ocupa desde hace unos años; precisamente en estudios anteriores (Falcón, V.L. 2011) hemos analizado la situación de las mujeres en el mercado laboral en la provincia del Chaco en 2001 observando el comportamiento de algunos indicadores que dieran cuenta de la condición general de varones y mujeres en el mercado laboral de la provincia.

${ }^{4}$ Nuestra principal fuente de datos es el Instituto Nacional de Estadística y Censos (INDEC) a partir de los Censos Nacionales de Población, Hogares y Vivienda 2001 y 2010 (Cuadro 9.9 Provincia de Chaco. Población de 14 años o más ocupada segun sexo y rama de actividad económica agrupada. Año 2001 y Cuadro P65-P. Provincia del Chaco. Población de 14 años y más ocupada por categoría ocupacional, según sexo y rama de actividad económica agrupada. 2010)

${ }^{5}$ Ver por ejemplo Escarrer, Catalina y otros. En: http://ve.umh.es/sieg.1/docs/lCongresolnternacional/comunicaciones/sciv06.pdf

${ }^{6}$ Para el año 2001 y 2010 la información sobre rama de actividad económica fue codificada de acuerdo al Clasificador de Actividades Económicas para Encuestas Socio demográficas del Mercosur,
}

Publicado en formato digital: Prof. Mgtr. Vilma Lilian Falcón. HACIA EL RECONOCIMIENTO DE LAS DESIGUALDADES ENTRE MUJERES Y VARONES EN EL MERCADO LABORAL DEL CHACO. EL COMPORTAMIENTO DE LA SEGREGACION HORIZONTAL EN LOS AÑOS 2001-2010. Revista Geográfica Digital. IGUNNE. Facultad de Humanidades. UNNE. Año 12. № 24. Julio - Diciembre 2015. ISSN 1668-5180 Resistencia, Chaco.

En: http://hum.unne.edu.ar/revistas/geoweb/default.htm 
siguiendo para el año 2001 la clasificación CAES Mercosur (18 ramas de actividades agrupadas) y para el año 2010 la versión CAES Mercosur. v1 (22 ramas de actividades). Cfr.

http://www.indec.gov.ar/ftp/cuadros/menusuperior/eph/caes_mercosur_1.0.pdf

${ }^{7} \mathrm{La}$ definición de este rubro es más compleja, es por êllo que se transcriben algunas de las descripciones que permiten comprender mejor el tipo de actividad. Respecto del apartado Actividades de los hogares en calidad de empleadores de personal doméstico: "incluye: las actividades de hogares que emplean personal doméstico, tales como criadas, cocineros, camareros, valets, mayordomos, lavanderos, jardineros, porteros, encargado de las caballerizas, conductores, vigilantes, institutriz, niñeras, profesores particulares, secretarias, etc. Esto permite al personal doméstico indicar la actividad de su patrón en censos o estudios, aunque el patrón sea un individuo. El producto generado por esta actividad es consumido por las casas de empleo" Por su parte, las Actividades indiferenciadas de producción de bienes de los hogares privados para uso propio hacen referencia a "actividades de hogares de producción de bienes de subsistencia indiferenciados, es decir las actividades de hogares involucradas en una variedad de actividades que producen bienes para su propia ubsistencia. Estas actividades incluyen caza y recolección, agricultura, la producción e refugio y ropa y otros bienes producidos por hogares para su propia subsistencia. Por otro lado, las Actividades indiferenciadas de producción de servicios de los hogares privados para uso propio refieren a "Los servicios en hogares para su propia subsistencia, es decir actividades de hogares que involucran una variedad de actividades que producen servicios para su propia subsistencia. Estas actividades incluyen cocinar, enseñar, cuidado de los miembros de la casa y otros servicios producidos por la casa para su propia subsistencia". Para ampliar: http://www.contraloria.gob.pa/inec/archivos/P4411T.pdf.-

${ }^{8}$ La elección del valor del $70 \%$ como línea de corte responde simplemente a la consideración de que una proporción de esa naturaleza no deja dudas de una marcada concentración.

${ }_{9}$ Para determinar que ocupaciones pueden calificarse como "femeninas", "masculinas" ó "integradas en término de género", hemos tomado la propuesta de Cáceres Ruiz, Juan y otros(2004) quienes, a partir del resultado que arroja el índice de Representación, consideran un umbral de 1,25 para definir las tres agrupaciones (ocupaciones femeninas, ocupaciones masculinas y ocupaciones integradas)

\section{Referencias Bibliográficas}

- Cáceres Ruiz, Juan Ignacio; Escot Mangas, Lorenzo; Fernández, José Andrés y Saiz Briones, Javier: (2004): "La segregación ocupacional y sectorial de la mujer en el mercado de trabajo español". Documento de trabajo. Universidad Complutense de Madrid. Disponible en: http://eprints.ucm.es/6830/1/0406.pdf

- Escarrer, Catalina y otros. La segregación horizontal y vertical por género entre el PAS y el PDI de la universitat de les Illes Balears. Consultado en: http://ve.umh.es/sieg.1/docs/ICongresolnternacional/comunicaciones/sciv06.pdf

- Falcón, Vilma L. (2011). Segregación ocupacional y sectorial de la mujer en el mercado de trabajo de la provincia del Chaco a inicios del siglo XXI. En: Investigaciones y Ensayos Geográficos. Revista de Geografía de la Universidad Nacional de Formosa. Año IX № 9.Formosa, Argentina.

- López, Guadalupe (2006). Segregación del mercado de trabajo en Argentina: Un abordaje de los cambios ocurridos en la relación entre educación y distribución del ingreso desde la perspectiva de género. Estudio para el período 1998- 2003. CLACSO. Buenos Aires, Argentina. En: http://bibliotecavirtual.clacso.org.ar/clacso/becas/20110206073138/lopez.pdf

- Maldonado, Victor y Teresa Rendón (2005) "Evolución reciente del trabajo de hombres y mujeres en México" Comercio Exterior, Vol 55, Núm 1, enero.

Publicado en formato digital: Prof. Mgtr. Vilma Lilian Falcón. HACIA EL RECONOCIMIENTO DE LAS DESIGUALDADES ENTRE MUJERES Y VARONES EN EL MERCADO LABORAL DEL CHACO. EL COMPORTAMIENTO DE LA SEGREGACION HORIZONTAL EN LOS AÑOS 2001-2010. Revista Geográfica Digital. IGUNNE. Facultad de Humanidades. UNNE. Año 12. № 24. Julio - Diciembre 2015. ISSN 1668-5180 Resistencia, Chaco.

En: http://hum.unne.edu.ar/revistas/geoweb/default.htm 
- Martinez Herrero, María José. Las Mujeres y la segregación laboral en la Unión Europea. Universidad del País Vasco. Disponible en: www.upo.es/congresos/export/sites/.../Mjose_Martinez.pdf

- Maté García, Jorge Julio; Nava Antolín, Luis Angel; Rodríguez Caballero, Juan Carlos. La segregación ocupacional por razón de género en Castilla y León. Departamento de Fundamentos del Análisis Económico. Universidad de Valladolid. España. Disponible en: www.jcyl.es/jcyl/cee/dgeae/congresos_ecoreg/CERCL/322.PDF

- Maté, J. J., Nava, A. y Rodríguez, J. C. (2002): "La segregación ocupacional por razón de sexo en la economía española, 1994-1999"; Revista del Ministerio de Trabajo y Asuntos Sociales, 36.

- Montero González, Bárbara; Hernández Peinado, Manuel y Camacho Ballesta, Jose A. Mercado de trabajo y juventud: análisis de la segregación laboral. En:

https://www.uam.es/otros/jaeet13/comunicaciones/11 Segregacion laboral/Montero Hernand ez Camacho.pdf

- Organización Internacional del Trabajo (OIT), 2014. Iniciativa del centenario de la OIT sobre las mujeres en el trabajo - dónde estamos, cuáles son nuestras metas. Mesa redonda conmemorativa del Día Internacional de la Mujer. Sede de la OIT, Ginebra. Suiza.

- Salas, Carlos y Leite, Marcia (2007) Segregación ocupacional por género: Una comparación Brasil-México. Colegio de Tlaxcala, México y Facultad de Educación, Universidade Estadual de Campinas. Disponible en: www.fcc.org.br/seminario/LEITE_SALAS.pdf

Publicado en formato digital: Prof. Mgtr. Vilma Lilian Falcón. HACIA EL RECONOCIMIENTO DE LAS DESIGUALDADES ENTRE MUJERES Y VARONES EN EL MERCADO LABORAL DEL CHACO. EL COMPORTAMIENTO DE LA SEGREGACION HORIZONTAL EN LOS AÑOS 2001-2010. Revista Geográfica Digital. IGUNNE. Facultad de Humanidades. UNNE. Año 12. № 24. Julio - Diciembre 2015. ISSN 1668-5180 Resistencia, Chaco.

En: http://hum.unne.edu.ar/revistas/geoweb/default.htm 
Revista Geográfica Digital. IGUNNE. Facultad de Humanidades. UNNE. Año 12. № 24. Julio - Diciembre 2015. ISSN 1668-5180 Resistencia, Chaco

\section{ANEXO}

Cuadro № 1. Provincia del Chaco. Ramas de Actividad Económica. Años 2001 y 2010

\section{SECTOR}

ECONOMICO

\section{RAMAS DE ACTIVIDAD}

2010
2001

\begin{tabular}{|c|c|c|}
\hline$\frac{\circ}{\frac{0}{\frac{2}{2}}}$ & $\begin{array}{l}\text { (A) Agricultura, ganadería, caza, silvicultura y pesca } \\
\text { (B) Explotación de minas y canteras }\end{array}$ & $\begin{array}{l}\text { A - Agricultura, ganadería, caza y silvicultura } \\
\text { B - Pesca y servicios conexos } \\
\text { C - Explotación de minas y canteras }\end{array}$ \\
\hline 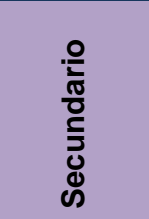 & $\begin{array}{l}\text { (C) Industria manufacturera } \\
\text { (D) Suministro de electricidad, gas, vapor y aire acor } \\
\text { (E) Suministro de agua; alcantarillado, gestión de } \\
\text { actividades de saneamiento } \\
\text { (F) Construcción }\end{array}$ & $\begin{array}{l}\text { D - Industria manufacturera } \\
\text { E - Electricidad, gas y agua } \\
\text { F - Construcción }\end{array}$ \\
\hline Terciario & $\begin{array}{l}\text { (G) Comercio al por mayor y al por menor; reparacio } \\
\text { automotores y motocicletas } \\
\text { (H) Transporte y almacenamiento } \\
\text { (I) Alojamiento y servicios de comidas } \\
\text { (J) Información y comunicación } \\
\text { (K) Actividades financieras y de seguros } \\
\text { (L) Actividades inmobiliarias } \\
\text { (M) Actividades profesionales, científicas y técnicas } \\
\text { (N) Actividades administrativas y servicios de apoyo } \\
\text { (O) Administración pública y defensa; planes de seg } \\
\text { obligatorio } \\
\text { (P) Enseñanza } \\
\text { (Q) Salud humana y servicios sociales } \\
\text { (R) Artes, entretenimiento y recreación } \\
\text { (S) Otras actividades de servicios } \\
\text { (T) Actividades de los hogares como empleadores d } \\
\text { doméstico; actividades de los hogares como produc } \\
\text { o servicios para uso propio } \\
\text { (U) Actividades de organizaciones y organismos extr }\end{array}$ & $\begin{array}{l}\text { G - Comercio al por mayor y menor; } \\
\text { reparación de vehículos automotores, } \\
\text { motocicletas, efectos personales y enseres } \\
\text { domésticos } \\
\text { H- Servicios de hotelería y restaurantes } \\
\text { I - Servicio de transporte, de } \\
\text { almacenamiento y de comunicaciones } \\
\text { J - Intermediación financiera y otros } \\
\text { servicios financieros } \\
\text { K - Servicios inmobiliarios, empresariales y } \\
\text { de alquiler } \\
\text { L - Administración pública, defensa y } \\
\text { seguridad social obligatoria } \\
\text { M - Enseñanza } \\
\mathrm{N} \text { - Servicios sociales y de salud } \\
\text { O - Servicios comunitarios, sociales y } \\
\text { personales } \\
\text { P - Servicios de hogares privados que } \\
\text { contratan servicio doméstico } \\
\text { Q - Servicios de organizaciones y órganos } \\
\text { extraterritoriales }\end{array}$ \\
\hline & (Z) Rama de actividad ignorada & Z - Actividades no bien especificadas \\
\hline
\end{tabular}

Fuente: Elaborado en base a INDEC. CNPHyV 2001 - 2010

Publicado en formato digital: Prof. Mgtr. Vilma Lilian Falcón. HACIA EL RECONOCIMIENTO DE LAS DESIGUALDADES ENTRE MUJERES Y VARONES EN EL MERCADO LABORAL DEL CHACO. EL COMPORTAMIENTO DE LA SEGREGACION HORIZONTAL EN LOS AÑOS 2001-2010. Revista Geográfica Digital. IGUNNE. Facultad de Humanidades. UNNE. Año 12. № 24. Julio - Diciembre 2015. ISSN 1668-5180 Resistencia, Chaco.

En: http://hum.unne.edu.ar/revistas/geoweb/default.htm 
Revista Geográfica Digital. IGUNNE. Facultad de Humanidades. UNNE. Año 12. № 24. Julio - Diciembre 2015. ISSN 1668-5180 Resistencia, Chaco

Cuadro N² . Participación de Mujeres y Varones ocupados según rama de actividad 2001

\begin{tabular}{|l|r|r|}
\hline \multicolumn{1}{|c|}{ Rama de Actividad Agrupada - 2001 } & Varones (\%) & Mujeres (\%) \\
\hline P - Servicios de hogares privados que contratan servicio doméstico & 9,70 & 90,30 \\
\hline M - Enseñanza & 26,19 & 73,81 \\
\hline N - Servicios sociales y de salud & 35,22 & 64,78 \\
\hline Q - Servicios de organizaciones y órganos extraterritoriales & 50,00 & 50,00 \\
\hline H - Servicios de hotelería y restaurantes & 53,31 & 46,69 \\
\hline Z - Actividades no bien especificadas & 58,53 & 41,47 \\
\hline O - Servicios comunitarios, sociales y personales n.c.p. & 62,42 & 37,58 \\
\hline K - Servicios inmobiliarios, empresariales y de alquiler & 69,69 & 30,31 \\
\hline L - Administración pública, defensa y seguridad social obligatoria & 69,71 & 30,29 \\
\hline J - Intermediación financiera y otros servicios financieros & 72,09 & 27,91 \\
\hline G - Comercio al por mayor y menor; reparación de vehículos automotores, motocicletas, efectos personale & 72,67 & 27,33 \\
\hline D - Industria manufacturera & 85,03 & 14,97 \\
\hline I - Servicio de transporte, de almacenamiento y de comunicaciones & 91,99 & 8,01 \\
\hline E - Electricidad, gas y agua & 92,12 & 7,88 \\
\hline A - Agricultura, ganadería, caza y silvicultura & 94,67 & 5,33 \\
\hline C - Explotación de minas y canteras & 96,15 & 3,85 \\
\hline B - Pesca y servicios conexos & 97,70 & 2,30 \\
\hline F - Construcción & 98,05 & 1,95 \\
\hline
\end{tabular}

Cuadro $N^{\circ} 3$. Participación de Mujeres y Varones ocupados según rama de actividad 2010

\begin{tabular}{|l|r|r|}
\hline \multicolumn{1}{|c|}{ Rama de actividad Agrupada - 2010 } & Varones (\%) & Mujeres (\%) \\
\hline (T) Actividades de los hogares como empleadores de personal doméstico; actividades de los hogares como & 20,14 & 79,86 \\
\hline (S) Otras actividades de servicios & 26,74 & 73,26 \\
\hline (P) Enseñanza & 35,60 & 64,40 \\
\hline Q) Salud humana y servicios sociales & 43,52 & 56,48 \\
\hline (I) Alojamiento y servicios de comidas & 47,87 & 52,13 \\
\hline (K) Actividades financieras y de seguros & 51,11 & 48,89 \\
\hline (M) Actividades profesionales, científicas y técnicas & 57,19 & 42,81 \\
\hline (G) Comercio al por mayor y al por menor; reparación de vehículos automotores y motocicletas & 58,34 & 41,66 \\
\hline (L) Actividades inmobiliarias & 59,31 & 40,69 \\
\hline (B) Explotación de minas y canteras & 60,69 & 39,31 \\
\hline (N) Actividades administrativas y servicios de apoyo & 61,50 & 38,50 \\
\hline (O) Administración pública y defensa; planes de seguro social obligatorio & 61,66 & 38,34 \\
\hline (R) Artes, entretenimiento y recreación & 62,83 & 37,17 \\
\hline (J) Información y comunicación & 66,39 & 33,61 \\
\hline (D) Suministro de electricidad, gas, vapor y aire acondicionado & 78,19 & 21,81 \\
\hline (C) Industria manufacturera & 80,53 & 19,47 \\
\hline (A) Agricultura, ganadería, caza, silvicultura y pesca & 82,80 & 17,20 \\
\hline (E) Suministro de agua; alcantarillado, gestión de desechos y actividades de saneamiento & 82,81 & 17,19 \\
\hline (Z) Rama de actividad ignorada & 86,08 & 13,92 \\
\hline (H) Transporte y almacenamiento & 88,72 & 11,28 \\
\hline
\end{tabular}

Fuente: Elaborado en base a INDEC. CNPHyV 2001 - 2010

Publicado en formato digital: Prof. Mgtr. Vilma Lilian Falcón. HACIA EL RECONOCIMIENTO DE LAS DESIGUALDADES ENTRE MUJERES Y VARONES EN EL MERCADO LABORAL DEL CHACO. EL COMPORTAMIENTO DE LA SEGREGACION HORIZONTAL EN LOS AÑOS 2001-2010. Revista Geográfica Digital. IGUNNE. Facultad de Humanidades. UNNE. Año 12. № 24. Julio - Diciembre 2015. ISSN 1668-5180 Resistencia, Chaco.

En: http://hum.unne.edu.ar/revistas/geoweb/default.htm 
Revista Geográfica Digital. IGUNNE. Facultad de Humanidades. UNNE. Año 12. № 24.

Julio - Diciembre 2015. ISSN 1668-5180 Resistencia, Chaco

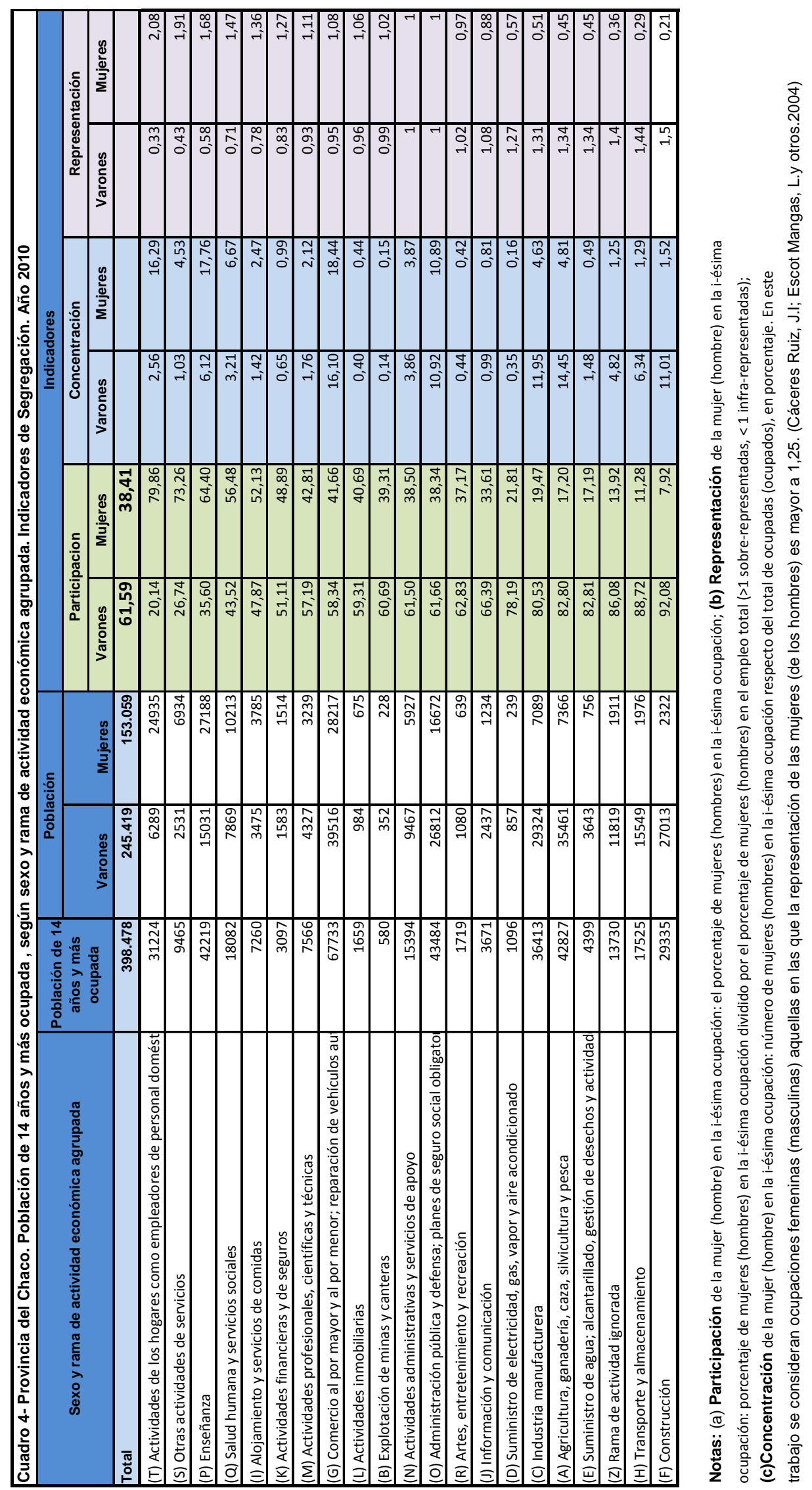

Publicado en formato digital: Prof. Mgtr. Vilma Lilian Falcón. HACIA EL RECONOCIMIENTO DE LAS DESIGUALDADES ENTRE MUJERES Y VARONES EN EL MERCADO LABORAL DEL CHACO. EL COMPORTAMIENTO DE LA SEGREGACION HORIZONTAL EN LOS AÑOS 2001-2010. Revista Geográfica Digital. IGUNNE. Facultad de Humanidades. UNNE. Año 12. № 24. Julio - Diciembre 2015. ISSN 1668-5180 Resistencia, Chaco.

En: http://hum.unne.edu.ar/revistas/geoweb/default.htm 
Revista Geográfica Digital. IGUNNE. Facultad de Humanidades. UNNE. Año 12. № 24. Julio - Diciembre 2015. ISSN 1668-5180 Resistencia, Chaco

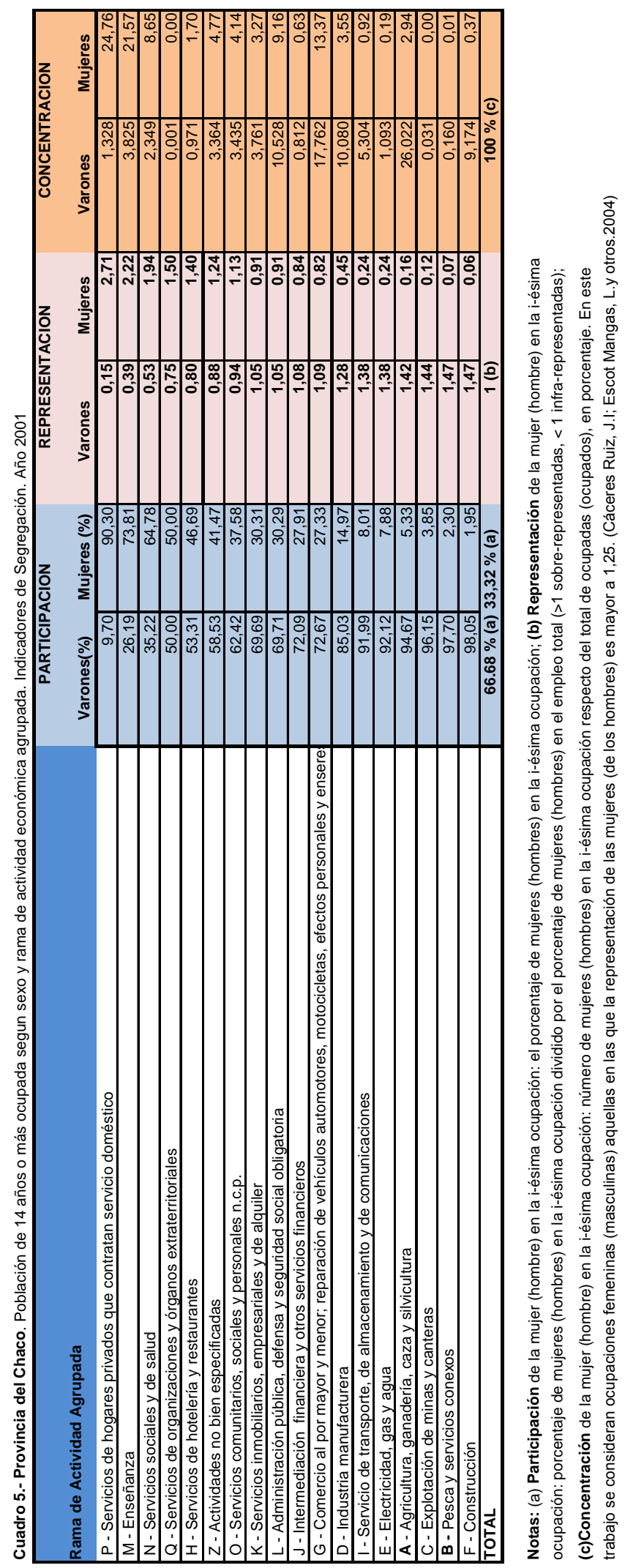

Publicado en formato digital: Prof. Mgtr. Vilma Lilian Falcón. HACIA EL RECONOCIMIENTO DE LAS DESIGUALDADES ENTRE MUJERES Y VARONES EN EL MERCADO LABORAL DEL CHACO. EL COMPORTAMIENTO DE LA SEGREGACION HORIZONTAL EN LOS AÑOS 2001-2010. Revista Geográfica Digital. IGUNNE. Facultad de Humanidades. UNNE. Año 12. № 24. Julio - Diciembre 2015. ISSN 1668-5180 Resistencia, Chaco.

En: http://hum.unne.edu.ar/revistas/geoweb/default.htm 